\title{
Proof of Principle of a Brain-Computer Interface Approach to Support Poststroke Arm Rehabilitation in Hospitalized Patients: Design, Acceptability, and Usability
}

\author{
Giovanni Morone, MD, ${ }^{a, b}$ Iolanda Pisotta, PsyD, ${ }^{c}$ Floriana Pichiorri, MD, ${ }^{a}$ \\ Sonja Kleih, PhD, ${ }^{d}$ Stefano Paolucci, MD, ${ }^{b}$ Marco Molinari, MD, PhD, ${ }^{c}$ \\ Febo Cincotti, PhD, ${ }^{a}$ e Andrea Kübler, PhD, ${ }^{d}$ Donatella Mattia, MD, $\mathrm{PhD}^{\mathrm{a}}$
}

From the ${ }^{a}$ Neuroelectrical Imaging and BCI Laboratory, Santa Lucia Foundation IRCCS, Rome, Italy; ${ }^{b}$ Clinical Laboratory of Experimental Neurorehabilitation, Santa Lucia Foundation IRCCS, Rome, Italy; ' Experimental Neurorehabilitation Laboratory, Santa Lucia Foundation IRCCS, Rome, Italy; ${ }^{d}$ Institute of Psychology, University of Würzburg, Würzburg, Germany; and ${ }^{e}$ Department of Computer, Control, and Management Engineering, Sapienza University of Rome, Italy.

\begin{abstract}
Objective: To evaluate the feasibility of brain-computer interface (BCI)-assisted motor imagery training to support hand/arm motor rehabilitation after stroke during hospitalization.

Design: Proof-of-principle study.

Setting: Neurorehabilitation hospital.

Participants: Convenience sample of patients $(\mathrm{N}=8)$ with new-onset arm plegia or paresis caused by unilateral stroke.

Interventions: The BCI-based intervention was administered as an "add-on" to usual care and lasted 4 weeks. Under the supervision of a therapist, patients were asked to practice motor imagery of their affected hand and received as a discrete feedback the movements of a "virtual" hand superimposed on their own. Such a BCI-based device was installed in a rehabilitation hospital ward.

Main Outcome Measures: Following a user-centered design, we assessed system usability in terms of motivation, satisfaction (by means of visual analog scales), and workload (National Aeronautics and Space Administration-Task Load Index). The usability of the BCI-based system was also evaluated by 15 therapists who participated in a focus group.

Results: All patients successfully accomplished the BCI training. Significant positive correlations were found between satisfaction and motivation $(P=.001, \mathrm{r}=.393)$. BCI performance correlated with interest $(P=.027, \mathrm{r}=.257)$ and motivation $(P=.012, \mathrm{r}=.289)$. During the focus group, professionals positively acknowledged the opportunity offered by BCI-assisted training to measure patients' adherence to rehabilitation. Conclusions: An ecological BCI-based device to assist motor imagery practice was found to be feasible as an add-on intervention and tolerable by patients who were exposed to the system in the rehabilitation environment.
\end{abstract}

Archives of Physical Medicine and Rehabilitation 2015;96(3 Suppl 1):S71-8

(C) 2015 by the American Congress of Rehabilitation Medicine

Stroke is a major cause of chronically impaired arm function among adults that may affect many activities of daily living. ${ }^{1}$ The

Presented to the National Institutes of Health, National Science Foundation, and other organizations (for a full list, see http://bcimeeting.org/2013/sponsors.html), June 3-7, 2013, Asilomar Conference Grounds, Pacific Grove, CA.

Supported in part by the European ICT Programme (project FP7-224631) (TOBI) and by the Italian Ministry of Health (grant no. RF-2010-2319611). This article only reflects the authors' views, and funding agencies are not liable for any use that may be made of the information contained herein.

Disclosures: none. standard rehabilitative approaches are still limited in terms of repetition, frequency, timing, and sensorimotor integration related to motor relearning. ${ }^{2,3}$ All of these factors are relevant in promoting the compensatory functional brain network reorganization associated with motor functional recovery in the acute, subacute, and chronic poststroke stage. ${ }^{4,5}$

Novel rehabilitative interventions have been proposed to assist task-specific repetition, such as active forms of robot-assisted upper limb therapy. ${ }^{6,7}$ In addition, patients' active involvment 
rewarded with performance-dependent feedback has been shown to be crucial in improving patient compliance-adherence to a given task-specific training. ${ }^{8}$ Also, evidence exists that the patient's level of participation in rehabilitation has an impact on the outcome. ${ }^{9}$

In severely affected hospitalized patients with little or no residual movements, the following worst-case scenario may occur: the patient is unable to perform active or assistive exercises and is unable to spend adequate time in rehabilitation activity during recovery, ${ }^{10,11}$ resulting in a drastically limited recovery potential. Consequently, there is an essential need for new, effective training strategies for stroke patients to match their specific needs and those of the rehabilitation professionals. ${ }^{12}$

Several authors have recently explored the potential of braincomputer interfaces (BCIs) for functional recovery after stroke. ${ }^{13,14}$ The BCI technology, based on volitional modulation of the electroencephalographic (EEG) sensorimotor rhythms (SMRs) in combination with motor imagery (MI) practice, ${ }^{15}$ robotic training, ${ }^{16,17}$ and functional electrical stimulation, ${ }^{18}$ has been recently promoted as a strategy to enhance motor recovery after stroke.

To effectively encourage training and practice, the BCI design should incorporate principles of current rehabilitative settings, apt to stimulate patients' engagement during a given exercise. This would be in line with recent prospective in BCI design, referred to as user-centered design, ${ }^{19}$ which drives the assistive technology solution implementation. ${ }^{20,21}$

In this proof-of-concept study, we report on an EEG-based BCI system intended to support hand MI training. Our system was designed in collaboration with professional users (ie, rehabilitation specialists and therapists) to reinforce the patients' participation in this task-specific training. As such, the BCI system was endowed with a visual feedback mimicking movements of the patient's own hands to maintain consistency with the MI task, ${ }^{22-27}$ and it was eventually intended as an add-on tool to enhance hand motor functional recovery of hospitalized patients affected by stroke. The BCI-assisted training also included the presence of a therapist to guide the patient during the training sessions, and it was introduced into a conventional motor rehabilitation setting - the hospital gym facility.

In accordance with this user-centered approach, professional users were requested to participate in the evaluation of the proposed add-on BCI-assisted rehabilitation training. The intervention was tested on a small, selected hospitalized patient sample, admitted for rehabilitation treatment after stroke, in order to describe its acceptability and usability. Following a user-centered design, we considered this preliminary evaluation as crucial for a

\begin{tabular}{|rl|}
\hline List of abbreviations: \\
BCI & brain-computer interface \\
BI & Barthel Index \\
EEG & electroencephalographic \\
MCID & minimal clinically important difference \\
MI & motor imagery \\
NASA-TLX & National Aeronautics and Space Administration-Task \\
& Load Index \\
QCM & Questionnaire for Current Motivation \\
QUEST & Quebec User Evaluation of Satisfaction with assistive \\
& Technology \\
SMR & sensorimotor rhythm \\
VAS & visual analog scale \\
\hline
\end{tabular}

subsequent randomized controlled trial to test the efficacy of this $\mathrm{BCI}$-assisted hand MI training.

\section{Methods}

\section{Participants and clinical evaluation}

A convenience sample of 8 patients with stroke (mean age \pm SD, $60 \pm 10.9 y$ ) was recruited from a consecutive cohort admitted to Santa Lucia Foundation, Scientific Institute for Research Hospitalization and Health Care. The demographic and clinical characteristics of the patients are reported in table 1. The study protocol was approved by the local ethical board, and written informed consent was obtained from each patient. Inclusion criteria were as follows: (1) hemiplegia/hemiparesis caused by a first-ever unilateral stroke and (2) age between 18 and 80 years. Exclusion criteria were as follows: (1) previous cerebrovascular accidents; (2) concomitant chronic disabling pathologies; (3) severe arm spasticity $\left(<4\right.$ on Modified-Ashworth Scale ${ }^{28}$ ); and (4) severe cognitive decline (Mini-Mental State Examination score $<24^{29}$ ). The arm-section of the Fugl-Meyer ${ }^{30}$ was adopted to describe intervention-specific functional improvements. A minimal clinically important difference (MCID) was set at 7 points. ${ }^{31}$ Other clinical descriptors were adopted including the National Institutes of Health Stroke Scale ${ }^{32}$ and the Barthel Index (BI; MCID for BI set at 14). ${ }^{31,33}$ These outcomes of rehabilitation efficacy were evaluated by a blinded assessor at baseline and after training; however, because of the absence of a proper control condition, we report a descriptive, qualitative analysis. In line with the scope of the pilot study, the assessment of acceptability and usability was considered as the primary outcome. The adopted measures are described in a separate section below (see Acceptability and Usability Assessment section).

\section{$B C I$ design, signal acquisition, and training protocol}

Figure 1 illustrates a training session performed with the proposed EEG-based BCI system. Patients were seated on a comfortable chair (or directly on their wheelchair) with their hands and forearms resting on a desk upon which an adjustable forearm orthosis provided support. Customized software was implemented to provide patients with real-time feedback consisting of a visual representation of their own arms and hands. The software allowed the therapists to create an artificial reproduction of a given patient's hand and arm by adjusting a digitally created image in shape, color, and size to match as much as possible the real hand and arm of the patient. This eventually led to the illusion of the patient's real hand movement when the BCI was successfully controlled. The digital image was projected over the patient's real hands covered by a white blanket. To drive the 2 states of the "virtual hand" (ie, grasping or finger extension), the BCI system exploited the EEG SMRs modulation induced by the performance of hand MI of the same movements. ${ }^{34-36}$

The BCI2000 software platform ${ }^{\mathrm{a}}$ was used for real-time estimation and classification of the SMRs state modulation and to drive the instant BCI visual feedback (ie, a cursor motion on a screen) and the corresponding "virtual hand" action. The "virtual hand" was actuated through a User Datagram Protocol connection between the BCI2000 platform and the "virtual hand" custom software. ${ }^{b}$ As in a conventional rehabilitation setting, the patient was also supported by the presence of a therapist during the 
Table 1 Demographic characteristics, clinical characteristics, and training outcome of patient sample

\begin{tabular}{|c|c|c|c|c|c|c|c|c|c|c|c|c|c|}
\hline \multirow[b]{2}{*}{ Patient No. } & \multirow[b]{2}{*}{ Sex } & \multirow[b]{2}{*}{$\begin{array}{l}\text { Age } \\
\text { (y) }\end{array}$} & \multirow{2}{*}{$\begin{array}{l}\text { Time } \\
\text { From } \\
\text { Event } \\
\text { (wk) }\end{array}$} & \multirow[b]{2}{*}{$\begin{array}{l}\text { Lesion } \\
\text { Side }\end{array}$} & \multirow[b]{2}{*}{$\begin{array}{l}\text { Lesion } \\
\text { Type }\end{array}$} & \multicolumn{2}{|c|}{$\begin{array}{l}\text { Fugl-Meyer Arm } \\
\text { Section }\end{array}$} & \multicolumn{2}{|c|}{ NIHSS } & \multicolumn{2}{|c|}{ Barthel Index } & \multicolumn{2}{|c|}{ Control Features } \\
\hline & & & & & & Pre & Post & Pre & Post & Pre & Post & Channel & Frequency $(\mathrm{Hz})$ \\
\hline 1 & M & 58 & 32 & $\mathrm{R}$ & I & 11 & 14 & 9 & 8 & 65 & 70 & $\mathrm{Cpz}, \mathrm{Cp} 2$ & $14-16$ \\
\hline 2 & M & 72 & 20 & $\mathrm{~L}$ & I & 13 & 17 & 10 & 7 & 45 & $65^{*}$ & Cp3, Сp1 & $16-18,22-24$ \\
\hline 3 & M & 58 & 44 & $\mathrm{R}$ & $\mathrm{H}$ & 13 & 15 & 8 & 8 & 90 & 90 & $\mathrm{C} 4, \mathrm{Cp} 4$ & $10-12$ \\
\hline 4 & M & 41 & 65 & $\mathrm{R}$ & $\mathrm{H}$ & 9 & $17^{*}$ & 5 & 5 & 45 & $70^{*}$ & $\mathrm{C} 2, \mathrm{C} 4, \mathrm{Cp} 4$ & $22-24$ \\
\hline 5 & $\mathrm{~F}$ & 75 & 6 & $\mathrm{~L}$ & I & 31 & $58^{*}$ & 12 & 5 & 40 & $55^{*}$ & $\mathrm{C} 3, \mathrm{C} 5$ & $16-18$ \\
\hline 6 & M & 52 & 9 & $\mathrm{R}$ & I & 10 & $17^{*}$ & 7 & 5 & 75 & 85 & C2, Сp2 & $18-20$ \\
\hline 7 & M & 58 & 7 & $\mathrm{~L}$ & I & 7 & 11 & 12 & 8 & 50 & $70^{*}$ & $\mathrm{Cpz}, \mathrm{Cp} 1$ & $22-24$ \\
\hline 8 & $\mathrm{~F}$ & 66 & 12 & $\mathrm{R}$ & I & 17 & $37^{*}$ & 9 & 6 & 45 & 55 & $\mathrm{Cz}, \mathrm{Cp} 4, \mathrm{Cp} 6$ & $14-16$ \\
\hline Mean \pm SD & & & & & & $13.9 \pm 7.1$ & $23.3 \pm 16.1$ & $9.0 \pm 2.4$ & $6.5 \pm 1.4$ & $56.9 \pm 17.9$ & $70 \pm 12.5$ & & \\
\hline
\end{tabular}

NOTE. Arm section of the Fugl-Meyer scale ranges from 0 (most affected) to 66 (normal); NIHSS ranges from 0 (normal) to 42 (most affected); Barthel Index ranges from 0 (most affected) to 100 (normal). Electrodes location on the head: C, central; $p$, parietal; $z$, zero-line (midline).

Abbreviations: F, female; $\mathrm{H}$, hemorrhagic; I, ischemic; L, left; M, male; NIHSS, National Institutes of Health Stroke Scale; R, right.

* Achievement of the minimal clinically important difference.

BCI-based MI training session. Accordingly, the therapist was provided with continuous BCI feedback displayed on a separate screen (ie, the cursor motion; see fig 1) to monitor the patients' MI performance in order to either verbally reinforce their correct behavior or support them to improve the necessary brain activation so they could achieve a better performance. Training lasted 4 weeks, with 3 weekly sessions. Each training session comprised 4 to 8 runs (20 trials each run), depending on the patient's physical capacity, and lasted approximately 30 minutes (EEG cap montage time excluded). The EEG was recorded from a subset of 31 electrodes distributed over the scalp centroparietal regions. Electromyographic activity was continuously recorded through surface electrodes placed over the opponens pollicis and the extensor digitorum communis of both the unaffected and affected hands. The electromyographic signal was fed back to the therapist to allow for online monitoring of muscle relaxation and detection of possible gross movements of the patients' hands and forearms hidden by the blanket (see fig 1).

During the BCI training, patients were asked to perform only 1 task (MI of the paralyzed hand, grasping or finger extension in separate runs) contrasted with a baseline condition to allow the system for online recognition and feedback processing. Thus, the trial length included a constant baseline period of 4 seconds and a task period of maximally 10 seconds (if brain activation did not lead to successful BCI control before the end of the trial).

The EEG features to control the cursor motion during the BCI training (see below) were extracted from a screening session during which patients were asked to perform MI of grasping and finger extension of their own affected hand, in separate runs and alternately with rest periods in a random fashion ( $15 \pm 1$ task trials and $15 \pm 1$ rest trials). The patients were cued by a visual interface on a computer screen, and no feedback of performance was provided. ${ }^{27}$ Scalp EEG potentials during the screening session were collected from 61 positions (according to an extension of the 1020 International System), bandpass filtered between 0.1 and $70 \mathrm{~Hz}$, digitized at $200 \mathrm{~Hz}$, and amplified by a commercial EEG system. ${ }^{\circ}$

An offline analysis was performed on these screening data to compare EEG signals associated with rest trials to those associated with MI trials with the affected hand. EEG data were rereferenced to the common average reference (ie, the average signal on every electrode site is taken and used as a reference), ${ }^{37,38}$ divided into epochs of 1 second, and spectral analysis was performed by means of a maximum entropy algorithm with a resolution of $2 \mathrm{~Hz}$. Differently from the online processing, when the system only computes the few features relevant for BCI control, all possible features in a reasonable range (ie, $0-60 \mathrm{~Hz}$ in $2-\mathrm{Hz}$ bins) were extracted and analyzed simultaneously. ${ }^{39}$ A feature vector was extracted from each epoch and labeled according to the experimental condition (rest or MI). This vector was composed of the spectral amplitude at each frequency bin for each channel. By using all the epochs of the recording session, the determination coefficient $R^{2}$ (ie, the proportion of the total variance of the signal amplitude accounted for by target position) ${ }^{40}$ was calculated to determine significant differences in the values of each feature in the 2 conditions. At the end of this process, $R^{2}$ values were compiled in a channel-frequency matrix together with head topography and evaluated to identify the set of candidate features that separated best rest versus MI. Based on visual inspection of these maps, an expert clinical neurophysiologist identified the best channel/frequency feature combination among central and centroparietal electrodes distributed over the affected hemisphere that showed desynchronization (ie, a decrease in power spectrum amplitude) at frequency bands typical for the SMRs modulation (namely, in the alpha and beta range; $8-24 \mathrm{~Hz}$ ). ${ }^{34}$ The degree of desynchronization on such selected electrodes/frequencies determined the vertical velocity of the cursor on the therapist's screen during the BCI training (continuous BCI feedback; see fig 1). Once the cursor reached a target that was positioned in the upper part of the screen, the "virtual hand" was actuated according to the imagined movement (discrete BCI feedback to patients in successful trials; see fig 1). In other words, successful MI and therefore desynchronization of the SMRs power recorded from the ipsilesional electrodes led to a cursor control (visible only to the therapist) and was translated into the movement of the projected "virtual" hand (see fig 1). Performance was calculated as the percentage of correct trials per run. Chance level was estimated in no-control conditions (subject at rest, not engaged in any task), in which modulations of SMRs were due only to physiological variability. In this condition, the average and SD of the BCI transducer's output were estimated. Under the hypothesis of Gaussian distribution, a corrective factor was applied so that the cursor would hit the target in only $5 \%$ of the trials (false 


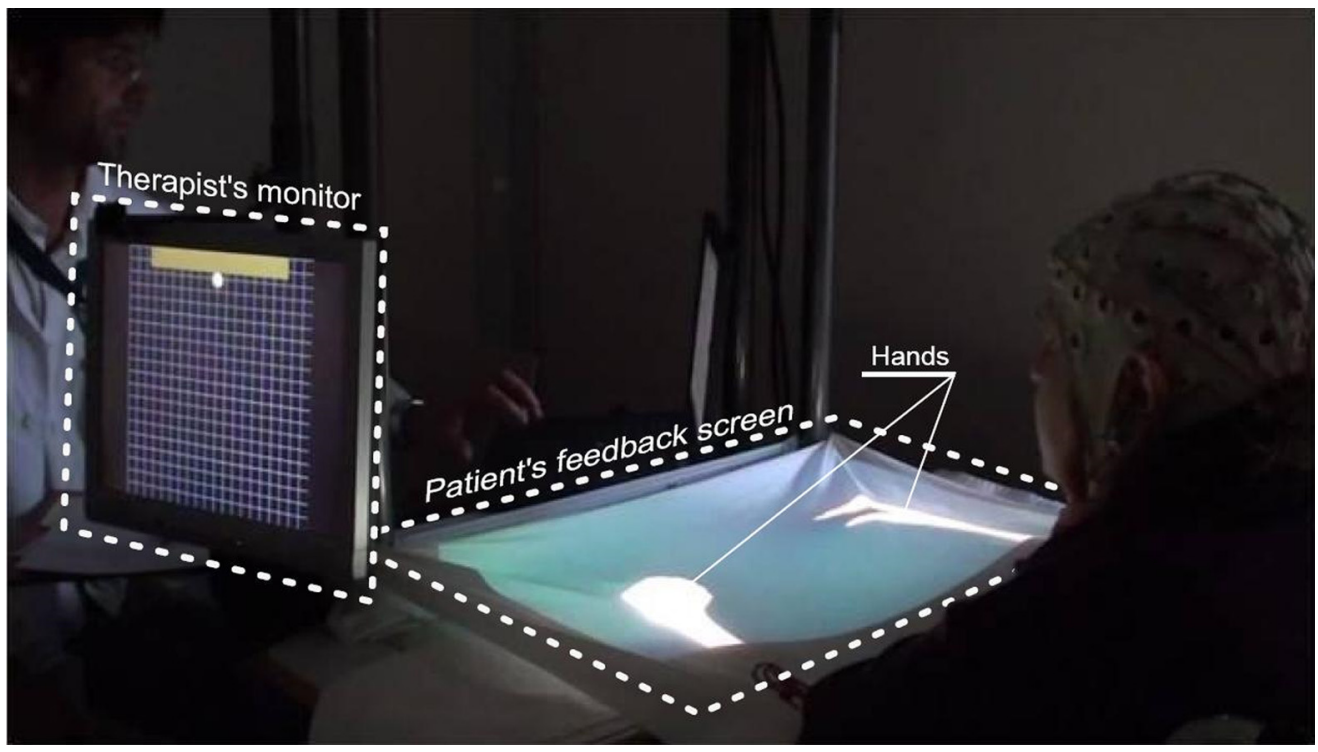

Fig 1 Training session with BCI EEG-based tool. In the training session, 2 actors take part: the patient and the therapist. The first is trained to gain control of his/her visual hand representation by imaging hand movements (grasping or finger extension), and he/she receives as feedback the congruent movements of the visual hand (successful trial). The therapist is fed back with the real-time movement of a cursor on a screen that is actually controlled by the patient BCI control feature.

positives). Empirical tests confirmed that after this gain correction, approximately 1 trial per run ended with an unintended hit.

\section{Acceptability and usability assessment}

Acceptability and usability were explored by means of participants' mood, motivation, and satisfaction assessment and participants' perceived workload. Usability was also assessed by professional users by means of a focus group setting.

Before starting each training session, patients' mood and motivation were monitored by means of visual analog scales (VASs; 0, the lowest level, to 10, the highest level). ${ }^{41,42}$ Mood was also assessed by means of the Center for Epidemiologic StudiesDepression Scale, ranging from 0 to 60 points, with a cutoff of 16 points above which individuals are considered at risk for clinical depression. ${ }^{43}$ To monitor such risk, the assessment was performed once a week across the 4 weeks of training. Motivation was also assessed by means of an adapted version of the Questionnaire for Current Motivation (QCM) ${ }^{44}$ which was administered at the end of each training session. The $\mathrm{QCM}^{45}$ is based on 4 motivational factors analyzed by means of 18 statements: (1) "mastery confidence," which refers to the certainty in succeeding at a task (4 statements); (2) "incompetence fear," indicating the level of anxiety about failing in the task (5 statements); (3) "challenge," denoting the perception of the task as a challenge (4 statements); and (4) "interest," which indicates how much the task may or may not evoke interest (5 statements). Each factor is scored as the averaged scores assigned to each statement belonging to that factor, ranging from 1 ("I completely disagree") to 7 ("I completely agree"). Satisfaction, defined as "freedom from discomfort and positive attitudes toward the use of the product," was reported by users by means of a VAS.

Workload is a hypothetical concept that represents the costs incurred by a human being to achieve a particular level of performance. ${ }^{20}$ The National Aeronautics and Space Administration-Task Load Index (NASA-TLX) has been proposed as an integrated measure of overall workload. It consists of 6 component subscales: time pressure, own performance, physical effort, mental effort, frustration, and stress and fatigue. The NASA-TLX was administered at the end of the first and last training session. As a measure of efficiency ${ }^{46}$ (ie, the invested costs in relation to how accurate and complete a task can be performed), the NASA TLX has proven its applicability in evaluation of BCI-controlled applications. $^{20,21}$ The NASA TLX was administered at the end of each training sessions.

The evaluation of the proposed BCI-based rehabilitation approach was also addressed with professional users identified as therapists in the context of a focus group. A focus group is a qualitative instrument frequently used in health services, which is particularly indicated when the participants are naive to the subject, and is meant to provide hints on unexpected ideas that might emerge from group discussion. ${ }^{47}$ Fifteen therapists attended a training session with the participation of 1 stroke patient. During this session, they were able to interact with both the patient and the experimenters. Afterward, therapists were administered a slightly modified version of the Quebec User Evaluation of Satisfaction with assistive Technology 2.0 (QUEST2.0), ${ }^{21,48}$ and an open discussion was held. The QUEST2.0 comprises 12 satisfaction items, with scoring ranging from 1 (very satisfied) to 5 (not satisfied at all).

\section{Statistical analysis}

To analyze changes in BCI performance, the second and last training sessions were considered for statistical analysis, conducted by means of a t test for dependent samples.

The Spearman coefficient was applied to explore separately the correlation between the BCI performance and each psychological variable and between psychological variables. The significance level was set at .05 .

\section{Results}

Table 1 reports the BCI control features (channel/frequency combination) for each patient. As mentioned above, BCI control 
Table 2 QCM scores relative to each of the 4 motivational factors (interest, challenge, mastery confidence, incompetence fear)

\begin{tabular}{lrrrrrrrrrr}
\hline Motivational & & & & & & & & & & \\
Factors & $S 1(n=8)$ & $S 2(n=8)$ & $S 3(n=8)$ & $S 4(n=8)$ & $S 5(n=8)$ & $S 6(n=8)$ & $S 7(n=6)$ & $S 8(n=6)$ & $S 9(n=6)$ & $S 10(n=5)$ \\
\hline Interest & $4.78 \pm 1.83$ & $4.56 \pm 1.41$ & $5.24 \pm 0.81$ & $5.68 \pm 0.98$ & $4.96 \pm 1.62$ & $5.08 \pm 1.76$ & $5 \pm 1.14$ & $4.88 \pm 1.90$ & $4.69 \pm 1.59$ & $5 \pm 1.83$ \\
Challenge & $4.34 \pm 1.90$ & $3.5 \pm 1.52$ & $3.41 \pm 1.77$ & $3.8 \pm 1.84$ & $4.23 \pm 2.06$ & $4.1 \pm 2.22$ & $3.74 \pm 2.65$ & $3.47 \pm 2.29$ & $3.18 \pm 2.86$ & $3.02 \pm 2.35$ \\
Mastery confidence & $5.56 \pm 0.83$ & $4.65 \pm 1.99$ & $5.40 \pm 1.37$ & $5.06 \pm 1.52$ & $5.46 \pm 1.61$ & $5.68 \pm 1.33$ & $5.70 \pm 1.62$ & $6.08 \pm 1.59$ & $6.05 \pm 1.07$ & $5.56 \pm 1.16$ \\
Incompetence fear & $4.35 \pm 1.66$ & $3.07 \pm 2.58$ & $3.92 \pm 2.44$ & $3.18 \pm 2.27$ & $3.32 \pm 2.31$ & $3.3 \pm 2.29$ & $3.4 \pm 2.46$ & $3.6 \pm 2.32$ & $3.43 \pm 1.87$ & $4 \pm 2.55$ \\
\hline
\end{tabular}

NOTE. Values are mean \pm SD. Each value represents the mean value \pm SD obtained from the 8-patient sample for each training session (S).

features were selected with constraints in both spatial and frequency domains to reinforce the MI- related brain activity by means of the BCI training. All patients succeeded in controlling the "virtual hand" by practicing MI of grasping and finger extension of the affected hand, achieving a mean percentage of performance of $57 \pm 24 \% \quad(n=8$ patients; 73 training sessions; chance level of 5\%). No significant changes in performance were found between the second $(62.3 \% \pm 20.4 \%)$ and the last $(49.1 \% \pm$ $19.1 \%)$ BCI training sessions $(P>.05)$.

From a qualitative point of view, we observed a clinically relevant posttraining increment in the arm section of Fugl-Meyer scores (MCID) in 4 patients (patient nos. 4, 5, 6, and 8 in table 1). The MCID was also reached for the BI scores in 4 patients (patient nos. 2, 4, 5, and 7 in table 1 ).

The patient sample was not at risk of depression as indicated by an average score of $6.86 \pm 4.8$ on the Center for Epidemiologic Studies-Depression Scale ( $\mathrm{n}=8$ patients). Patients rated their mood as good (VAS: $7.15 \pm 1.91$, average of all sessions of all patients) and were highly motivated during training (VAS score mean: 7.70 \pm 1.90 , average of all sessions of all patients); satisfaction was also high (VAS score mean: $8.36 \pm 1.65$, average of all sessions of all patients). VAS mood and motivation scores positively correlated $(P=.00, \mathrm{r}=.479)$, and satisfaction was also positively correlated with motivation $(P=.001, \mathrm{r}=.393)$. As illustrated in table 2, the QCM mean scores relative to each factor and each training session had a tendency to vary up to the fifth training session. As the training proceeded, the "mastery confidence" showed an increasing trend, whereas the opposite was observed for the "challenge" factor. The "interest" and the "incompetence fear" factors were substantially stable, and they showed at the end of the training an overall increase and decrease, respectively. Furthermore, the "interest" QCM factor and the BCI performance percentage were positively correlated $(P=.027$,

Table 3 QUEST2.0: percentage of most important items and mean values of mentioned items of 15 physiotherapists

\begin{tabular}{lcc}
\hline $\begin{array}{l}\text { Items } \\
\text { (Satisfaction With) }\end{array}$ & $\begin{array}{l}\text { Percentage } \\
\text { of Importance }\end{array}$ & $\begin{array}{l}\text { Mean Score } \\
\text { (Min=0; } \text { Max =5) }\end{array}$ \\
\hline Effectiveness & 71 & 4 \\
Ease to use & 57 & 4 \\
Learnability & 57 & 4 \\
Safety & 42 & 4 \\
Reliability & 42 & 4 \\
\hline
\end{tabular}

NOTE. First and second columns show the most important satisfaction items in order of importance to be considered in a group of physiotherapists participating in the focus group. Third column shows mean values of satisfaction with the selected item.

Abbreviations: Max, maximum; Min, minimum. $\mathrm{r}=.257)$, as were the BCI performance and VAS motivation scores $(P=.012, \mathrm{r}=.289)$.

Analysis of the NASA-TLX questionnaires revealed that no significant differences in perceived workload were found between the overall mean values ( $n=8$ patients) obtained at the end of the first and last training sessions (NASA-TLX: 50.22 \pm 21.73 end of first session; $54.17 \pm 23$ end of last session).

According to the QUEST2.0 results, all therapists $(n=15)$ stated that the most important system features were "effectiveness," "ease to use," "learnability," "safety," and "reliability" (table 3).

Several strengths and weaknesses of the BCI-assisted MI training design emerged from the open discussion. Professionals identified as a strength the potentiality of such a BCI-based system to provide them with a quantitative measure of the patients' adherence to a cognitive-motor rehabilitation session (ie, SMR modulation induced by MI). They considered the most relevant weaknesses to be (1) the prototype setup and functioning (hardware and software), which require technical skills that "a therapist might not have," and they would not feel confident in being able to carry out a session without some technical assistance (cap and electrodes adjusting, EEG signal monitoring, software operating); (2) the lack of a "goal-directed action" feedback (eg, "holding and releasing a glass of water"); and (3) the need to monitor a possible increase in arm spasticity during MI task practice.

\section{Discussion}

The present pilot study described an EEG-based BCI tool designed to support arm/hand motor rehabilitation after stroke. The proposed EEG-based BCI tool was meant to support taskspecific training such as the imagery of simple hand movements (MI) in stroke patients with motor deficit. As such, the system was tested on a sample of stroke patients with severe motor impairment (see table 1) who were admitted to the hospital for their conventional rehabilitation treatment.

The design was inspired by some rehabilitation principles that included the presence of the therapist to guide the patient during the training sessions, and the introduction in a real conventional rehabilitation setting. Moreover, the visual feedback ("virtual hand") was implemented to match the MI task content ${ }^{44}$ in order to reinforce patients' participation in task performance. According to a user-centered design, we first undertook a study to evaluate the acceptability and usability of this ecological ${ }^{49}$ BCI-based system by both the patients and the professional users, namely the therapists.

A comprehensive psychological assessment data set was thus presented to evaluate system acceptability and usability. This latter aspect was also evaluated with the participation of professional end-users by means of instruments such as a focus group and a questionnaire, with the aim of further investigating the 
feasibility of the proposed intervention in a real rehabilitative realm. ${ }^{20}$ Differently, only a qualitative description of the clinical motor function indicators was reported, since the experimental treatment (ie, the BCI-assisted MI training) efficacy is beyond the scope of this pilot study.

Task-specific training has been demonstrated to engage neural plasticity ${ }^{50,51}$ in models of animal and human motor (re)-learning after stroke, and the combination of aerobic exercise and taskspecific training still remains the criterion standard treatment in poststroke rehabilitation. ${ }^{52}$ However, task-specific training repetitions performed in conventional therapy are still limited with respect to those required to impact on brain reorganizational changes related to activity-based therapies. ${ }^{53}$

Our preliminary data gathered on a limited sample of stroke patients suggest that BCI technology might successfully be adopted to support the practice of MI tasks and thus positively influence outcome measures of functional motor recovery in stroke patients. A clinical trial with a large cohort of patients is needed to establish the extent to which any clinical improvement might be imputed to the BCI technology supporting the MI training, and to confirm the current positive results on the acceptability of the system by patients. In this regard, a randomized controlled trial is currently in progress in our rehabilitation center to investigate the effects of the proposed EEG-BCI-based tool on functional motor scales and also on previously validated neurophysiological measures of brain plasticity. ${ }^{54}$

All patients were highly motivated, supporting the idea that the specific BCI training was positively accepted with a good compliance/adherence. In particular, we observed that motivation was maintained across training sessions, and motivation scores correlated with satisfaction in being exposed to the BCI system. Similarly, by the motivational factors derived from the QCM, the trend showed that patients exposed to the BCI-based training felt that this experience was doable, not overchallenging, and interesting. Compliance and adherence are very relevant in technology-supported therapy that might appear alienating for its intrinsic characteristics and might be perceived as disrupting in the patient-therapist relationship. ${ }^{55}$ This increases the need for active top-down involvement of patients, contrasting with the general bottom-up approach based primarily on passive mobilization. ${ }^{56}$ Our ecological BCI-based device was designed in the attempt to cope with this apparent gap. Patients were also motivated by an enriched visual feedback reinforcing the correct execution of the rehabilitation task. The importance of feedback was clearly shown in our results in which positive performance influenced the patients' satisfaction.

Our findings of a positive correlation between performance and motivation are in line with those of Kleih et al, ${ }^{57}$ who monitored motivation in a sample of healthy subjects during regulation of SMRs by means of MI. We speculate that positive mood and motivation might play a positive role in the BCI-based rehabilitation, strengthening patients' active involvement in our technology-assisted treatment, ${ }^{55}$ that in turn would lead to a higher top-down central nervous system engagement during taskspecific training. ${ }^{56}$

Encouragingly, we also found no significant self-rated workload differences across training. We noticed a different trend in the workload perceived by the patients when considering the time from stroke. In fact, an initially higher workload was experienced by patients with stroke in chronic phase with respect to what they reported at the end of the 1-month training. The opposite trend was observed in the patients with stroke in subacute phase. If confirmed on a large number of patients, this preliminary observation might well reflect the need of tailored ad hoc amount of exposure to a BCI-based intervention in different stages of poststroke recovery.

The acceptability of the proposed system by therapists highly depends on a subjective technical confidence and a positive attitude toward the use of technologies. Improvements are needed especially in regard to the EEG cap montage and the time needed to set up the system prototype. Usability was rated as high by patients and therapist. High learnability emerged in the focus group therapists, as did efficiency of the device, providing a high percentage of well-executed tasks with a considerable number of repetitions.

\section{Study limitations}

The small, heterogeneous sample size and the lack of a control condition are regarded as limitations that prevent drawing any definitive conclusions about the efficacy of the proposed intervention, thus limiting the current study to a feasibility study. As such, the current findings on acceptability and usability also require a further validation in a larger cohort of patients. To address these issues, a randomized controlled trial is currently in progress in our rehabilitation center.

\section{Conclusions}

This pilot study supports the feasibility of a specifically designed BCI-based training to support hand/arm motor rehabilitation after stroke. Our BCI-assisted MI training has proven to be tolerable and acceptable to patients, and although usability still requires some improvement, professional users are prone to accept such technology when added to standard motor rehabilitation during hospitalization.

The user-centered design contributes to the acceptability and usability of the proposed implemented BCI-based device, thus providing a background for investigating its clinical efficacy in real rehabilitative environments.

\section{Suppliers}

a. BCI2000 software platform. Available at: www.bci2000.org.

b. "Virtual hand" developed in the Neuroelectrical Imaging and BCI Laboratory, Santa Lucia Foundation, Rome.

c. BrainAmp; Brain Products GmbH.

\section{Keywords}

Brain-computer interfaces; Rehabilitation; Stroke

\section{Corresponding author}

Giovanni Morone, MD, Santa Lucia Foundation IRCCS, Via Ardeatina, 306-00179 Roma, Italy. E-mail address: g.morone@ hsantalucia.it.

\section{References}

1. Jørgensen HS, Nakayama H, Raaschou HO, Vive-Larsen J, Støier M, Olsen TS. Outcome and time course of recovery in stroke. Part II: time course of recovery. The Copenhagen Stroke Study. Arch Phys Med Rehabil 1995;76:406-12. 
2. Lang CE, Macdonald JR, Reisman DS, et al. Observation of amounts of movement practice provided during stroke rehabilitation. Arch Phys Med Rehabil 2009;90:1692-8.

3. Bastian AJ. Understanding sensorimotor adaptation and learning for rehabilitation. Curr Opin Neurol 2008;21:628-33.

4. Kwakkel G, Wagenaar RC, Twisk JW, Lankhorst GJ, Koetsier JC. Intensity of leg and arm training after primary middle-cerebral-artery stroke: a randomised trial. Lancet 1999;354:191-6.

5. Volpe BT, Lynch D, Rykman-Berland A, et al. Intensive sensorimotor arm training mediated by therapist or robot improves hemiparesis in patients with chronic stroke. Neurorehabil Neural Repair 2008;22:305-10.

6. Iosa M, Morone G, Fusco A, et al. Seven capital devices for the future of stroke rehabilitation. Stroke Res Treat 2012;2012:187965.

7. Lo AC, Guarino PD, Richards LG, et al. Robot-assisted therapy for long-term upper-limb impairment after stroke. N Engl J Med 2010; 362:1772-83.

8. Cirstea MC, Levin MF. Improvement of arm movement patterns and endpoint control depends on type of feedback during practice in stroke survivors. Neurorehabil Neural Repair 2007;21:398-411.

9. Paolucci S, Di Vita A, Massicci R, et al. Impact of participation on rehabilitation results: a multivariate study. Eur J Phys Rehabil Med 2012:48:455-66.

10. Bernhardt J, Dewey H, Thrift A, Donnan G. Inactive and alone: physical activity within the first 14 days of acute stroke unit care. Stroke 2004;35:1005-9.

11. King A, McCluskey A, Schurr K. The time use and activity levels of inpatients in a co-located acute and rehabilitation stroke unit: an observational study. Top Stroke Rehabil 2011;18(Suppl 1):654-65.

12. Barker RN, Brauer SG. Upper limb recovery after stroke: the stroke survivors perspective. Disabil Rehabil 2005;27:1213-23.

13. Silvoni S, Ramos-Murguialday A, Cavinato M, et al. Brain-computer interface in stroke: a review of progress. Clin EEG Neurosci 2011;42: 245-52.

14. Daly JJ, Wolpaw JR. Brain-computer interfaces in neurological rehabilitation. Lancet Neurol 2008;7:1032-43.

15. Prasad G, Herman P, Coyle D, McDonough S, Crosbie J. Applying a brain-computer interface to support motor imagery practice in people with stroke for upper limb recovery: a feasibility study. J Neuroeng Rehabil 2010;7:60.

16. Várkuti B, Guan C, Pan Y, et al. Resting state changes in functional connectivity correlate with movement recovery for BCI and robotassisted upper-extremity training after stroke. Neurorehabil Neural Repair 2013;27:53-62.

17. Ramos-Murguialday A, Broetz D, Rea M, et al. Brain-machine interface in chronic stroke rehabilitation: A controlled study. Ann Neurol 2013;74:100-8.

18. Do AH, Wang PT, King CE, Abiri A, Nenadic Z. Brain-computer interface controlled functional electrical stimulation system for ankle movement. J Neuroeng Rehabil 2011;8:49.

19. ISO DIS 9241-210:2008. Ergonomics of human system interactionpart 210: human-centred design for interactive systems (formerly known as 13407). Switzerland: International Standardization Organization (ISO). Available at: www.iso.org. Accessed March 15, 2012.

20. Riccio A, Leotta F, Bianchi L, et al. Workload measurement in a communication application operated through a P300-based braincomputer interface. J Neural Eng 2011;8:025028.

21. Zickler C, Riccio A, Leotta F, et al. A brain-computer interface as input channel for a standard assistive technology software. Clin EEG Neurosci 2011;42:236-44.

22. Vargas CD, Olivier E, Craighero L, Fadiga L, Duhamel JR, Sirigu A. The influence of hand posture on corticospinal excitability during motor imagery: a transcranial magnetic stimulation study. Cereb Cortex 2004; 14:1200-6.

23. de Vries S, Tepper M, Otten B, Mulder T. Recovery of motor imagery ability in stroke patients. Rehabil Res Pract 2011;2011: 283840.

24. Cicinelli P, Marconi B, Zaccagnini M, Pasqualetti P, Filippi MM, Rossini PM. Imagery-induced cortical excitability changes in stroke: a transcranial magnetic stimulation study. Cereb Cortex 2006;16: 247-53.

25. Sharma N, Simmons LH, Jones PS, et al. Motor imagery after subcortical stroke: a functional magnetic resonance imaging study. Stroke 2009; 40:1315-24.

26. Cattaneo L, Caruana F, Jezzini A, Rizzolatti G. Representation of goal and movements without overt motor behavior in the human motor cortex: a transcranial magnetic stimulation study. J Neurosci 2009;29:11134-8.

27. Kaiser V, Daly I, Pichiorri F, Mattia D, Müller-Putz GR, Neuper C. Relationship between electrical brain responses to motor imagery and motor impairment in stroke. Stroke 2012;43:2735-40.

28. Bohannon R, Smith M. Interrater reliability of a Modified Ashworth Scale of muscle spasticity. Phys Ther 1987;67:206.

29. Tombaugh TN. Test-retest reliable coefficients and 5-year change scores for the MMSE and 3MS. Arch Clin Neuropsychol 2005;20: 485-503.

30. Gladstone DJ, Danells CJ, Black SE. The Fugl-Meyer assessment of motor recovery after stroke: a critical review of its measurement properties. Neurorehabil Neural Repair 2002;16:232-40.

31. Sivan M, O’Connor RJ, Makower S, Levesley M, Bhakta B. Systematic review of outcome measures used in the evaluation of robotassisted upper limb exercise in stroke. J Rehabil Med 2011;43:181-9.

32. Goldstein LB, Bertels C, Davis JN. Interrater reliability of the NIH Stroke Scale. Arch Neurol 1989;46:660-2.

33. Mahoney FI, Barthel DW. Functional evaluation: the Barthel Index. Md State Med J 1965;14:61-5.

34. Pfurtscheller G, Lopes da Silva FH. Event-related EEG/MEG synchronization and desynchronization: basic principles. Clin Neurophysiol 1999;110:1842-57.

35. Neuper C, Scherer R, Reiner M, Pfurtscheller G. Imagery of motor actions: differential effects of kinesthetic and visual-motor mode of imagery in single-trial EEG. Brain Res Cogn Brain Res 2005;25: 668-77.

36. Pichiorri F, De Vico Fallani F, et al. Sensorimotor rhythm-based brain-computer interface training: the impact on motor cortical responsiveness. J Neural Eng 2011;8:025020.

37. Osselton JW. Acquisition of EEG data by bipolar, unipolar and average reference methods: a theoretical comparison. Electroencephalogr Clin Neurophysiol 1965;19:527-8.

38. Cooper R, Binnie CD, Osselton JW, Prior PF, Wisman T. EEG, paediatric neurophysiology, special techniques and applications. In: Cooper R, Mauguiere F, Osselton JW, Prior PF, Tedman BM, editors. Clinical neurophysiology, vol 2. Amsterdam: Elsevier; 2003. p 8-103.

39. Cincotti F, Mattia D, Aloise F, et al. Non-invasive brain-computer interface system: towards its application as assistive technology. Brain Res Bull 2008:796-803.

40. Cincotti F, Mattia D, Aloise F, et al. High-resolution EEG techniques for brain-computer interface applications. J Neurosci Methods 2008; $167: 31-42$.

41. Wewers ME, Lowe NK. A critical review of visual analogue scales in the measurement of clinical phenomena. Res Nurs Health 1990;13: 227-36.

42. Kleih SC, Nijboer F, Halder S, Kübler A. Motivation modulates the P300 amplitude during brain-computer interface use. Clin Neurophysiol 2010;121:1023-31.

43. Dozeman E, van Schaik DJ, van Marwijk HW, Stek ML, van der Horst HE, Beekman AT. The Center for Epidemiological Studies Depression Scale (CES-D) is an adequate screening instrument for depressive and anxiety disorders in a very old population living in residential homes. Int J Geriatr Psychiatry 2011;26:239-46.

44. Bunketorp Käll L, Lundgren-Nilsson $\AA$, Blomstrand C, Pekna M, Pekny M, Nilsson M. The effects of a rhythm and music-based therapy program and therapeutic riding in late recovery phase following stroke: a study protocol for a three-armed randomized controlled trial. BMC Neurol 2012;12:141.

45. Nijboer F, Birbaumer N, Kübler A. The influence of psychological state and motivation on brain-computer interface performance in 
patients with amyotrophic lateral sclerosis - a longitudinal study. Front Neurosci 2010;4:55.

46. NASA Human Performance Research Group, NASA Task Load Index (NASA-TLX). NASA Ames Research Center, NASA Human Performance Research Group; 1987. Available at: http:// humansystems.arc.nasa.gov/groups/TLX. Accessed March 1, 2011.

47. Kitzinger J. Qualitative research. Introducing focus groups. BMJ 1995;311:299-302.

48. Demers L, Weiss-Lambrou R, Ska B. Quebec User Evaluation of Satisfaction with assistive Technology. QUEST version 2.0. An outcome measure for assistive technology devices. Institute for Matching Person and Technology; New York: Webster; 2000.

49. Rouse WB. People and organizations: explorations of humancentered design. Hoboken: Wiley; 2007.

50. Nudo RJ. Mechanisms for recovery of motor function following cortical damage. Curr Opin Neurobiol 2006;16:638-44.

51. Murphy TH, Corbett D. Plasticity during stroke recovery: from synapse to behaviour. Nat Rev Neurosci 2009;10: 861-72.
52. Dobkin BH, Dorsch A. New evidence for therapies in stroke rehabilitation. Curr Atheroscler Rep 2013;15:331.

53. Lang CE, MacDonald JR, Gnip C. Counting repetitions: an observational study of outpatient therapy for people with hemiparesis poststroke. J Neurol Phys Ther 2007;31:3-10.

54. De Vico Fallani F, Pichiorri F, Morone G, et al. Multiscale topological properties of functional brain networks during motor imagery after stroke. Neuroimage 2013;83C:438-49.

55. Bragoni M, Broccoli $\mathrm{M}$, Iosa $\mathrm{M}$, et al. Influence of psychological features on rehabilitation outcomes in patients with subacute stroke trained with robotic-aided walking therapy: a controlled longitudinal observational pilot study. Am J Phys Med Rehabil 2013;92(10 Suppl 2):e16-25.

56. Belda-Lois JM, Mena-del Horno S, Bermejo-Bosch I, et al. Rehabilitation of gait after stroke: a review towards a top-down approach. J Neuroeng Rehabil 2011;8:66.

57. Kleih SC, Riccio A, Mattia D, et al. Motivation influences performance in SMR-BCI. Proceedings of the 5th International BrainComputer Interface Conference; 2011 Sep 22-24; Graz (Austria). Graz (Austria): Graz University of Technology; 2011. p 108-111. 